\title{
Subcostal nerve injury after laparoscopic lipoma surgery: an unusual culprit for an unusual complication
}

\author{
Hussam Abou-Al-Shaar, MD,, ${ }^{1,2}$ and Mark A. Mahan, MD' ${ }^{1}$ \\ 'Department of Neurosurgery, Clinical Neurosciences Center, University of Utah, Salt Lake City, Utah; and 2Department of \\ Neurosurgery, Hofstra Northwell School of Medicine, Manhasset, New York \\ Endoscopic surgery has revolutionized the field of minimally invasive surgery. Nerve injury after laparoscopic surgery \\ is presumably rare, with only scarce reports in the literature; however, the use of these techniques for new purposes \\ presents the opportunity for novel complications. The authors report a case of subcostal nerve injury after an anterior \\ laparoscopic approach to a posterior abdominal wall lipoma. \\ A 62-year-old woman presented with a left abdominal flank bulge (pseudohernia) that developed after laparoscopic \\ posterior flank wall lipoma resection. Imaging demonstrated frank ballooning of the oblique muscles; denervation atrophy \\ and thinning of the external oblique, internal oblique, and transverse abdominis muscles; and thinning of the rectus \\ abdominis muscle. The patient underwent subcostal nerve repair and removal of a foreign plastic material from the \\ laparoscopic procedure. At 8 months, she has regained substantial improvement in abdominal wall strength. \\ Although endoscopic procedures have resulted in significant reduction in morbidity, "minimally invasive" approaches \\ should not be confused with "low risk" when approaching novel pathology. The subcostal nerve is at risk of injury in \\ posterior abdominal wall surgery, whether laparoscopic or not. With the pseudohernia and abdominal bulge after this \\ surgery, the cosmetic appeal of laparoscopic incisions was definitively undone. Selecting an approach based on the \\ anatomy of adjacent structures may lead to a better functional result. \\ https://thejns.org/doi/abs/10.3171/2018.7.JNS18532
}

KEYWORDS subcostal nerve; T12; laparoscopy; complication; abdominal wall; pseudohernia; peripheral nerve

$\mathrm{L}$ APAROSCOPIC surgery has revolutionized the field of minimally invasive surgery. The employment of the laparoscope in abdominal, thoracic, and spinal surgery has resulted in significant reductions in hospital stay, pain, blood loss, and costs, and it has substantially improved patient satisfaction and aesthetic outcomes. . $^{4,5,12,13}$ However, advanced techniques and technology do not eliminate surgical complications, despite reduction in invasiveness. Rather, new complications can arise as a consequence of the use of such tools to address unique pathological conditions or in novel procedures. A new set of complications, such as organ perforation, vessel damage, and wound complications, was soon recognized for laparoscopic surgery. ${ }^{4,5,12,13}$ Nerve injury after laparoscopic surgery is extremely rare, with only scarce reports in the literature. ${ }^{2,5,6,10,14,16}$ Herein, we report an unusual case of subcostal nerve injury occurring after laparoscopic posterior flank wall lipoma resection, and we review the anatomy of the subcostal nerve and its relation to adjacent structures.

\section{Case Report}

History and Examination

A 62-year-old woman presented to our clinic complaining of a left abdominal flank bulge of 3 months' duration. She noted that the bulge developed progressively after an anterior laparoscopic approach for the resection of a posterior flank wall lipoma at an outside institution. The lipoma had been discovered incidentally by imaging. Otherwise, at the time of diagnosis of the lipoma, the patient was completely asymptomatic.

Immediately after her laparoscopic surgery, she reported excruciating pain radiating around the left abdominal flank. Although the severity of pain after surgery was noted to be unusual, she was discharged the same day. 


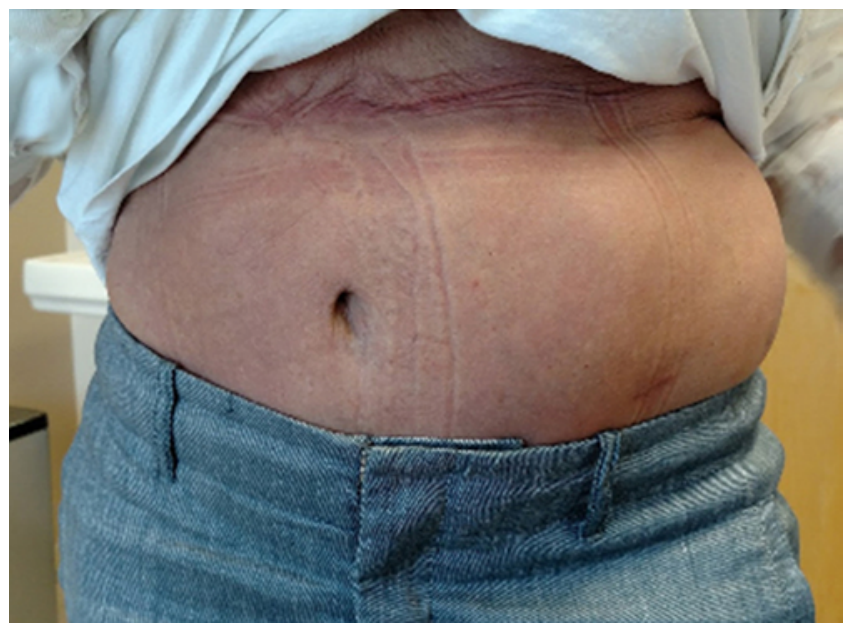

FIG. 1. Preoperative photograph depicting clear left-sided pseudohernia and abdominal bulge. Figure is available in color online only.

Following surgery, she noted progressive worsening of an abdominal wall paresis, with a frank flank bulge (pseudohernia). She also noted difficulty with core strength, particularly when she was trying to ride her mountain bike. She described dull hypesthesia on the left flank wall. Pain was no longer a predominant symptom.

On physical examination at our institution, there was an evident bulge on the left abdominal wall (Fig. 1). The muscle tone was flaccid in the left abdominal wall compared with the right side and without voluntary contractibility. Hypesthesia was also evident on the left flank wall on sensory examination. The rest of her examination was unremarkable.

Preoperative electromyography (EMG) showed no evidence of recruitable motor units in the oblique muscles or rectus abdominis muscles, indicative of unrecovered and severe axonotmesis.

\section{Imaging Studies}

A CT scan of the abdomen and pelvis prior to the patient's lipoma resection demonstrated a lipoma-like mass

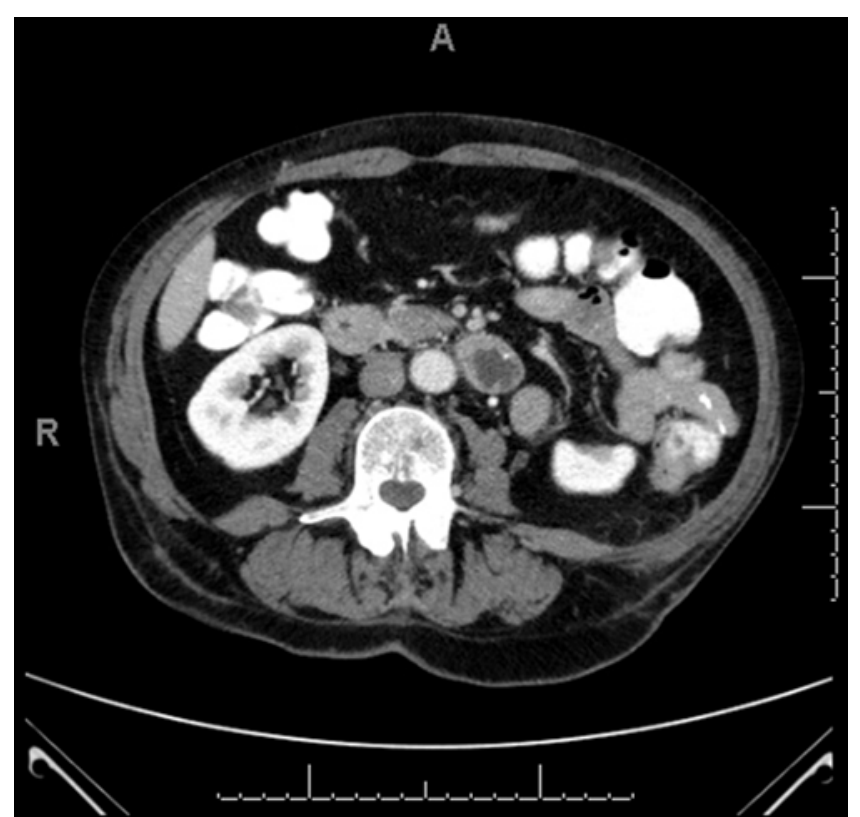

FIG. 3. Axial CT of the patient's abdomen after her lipoma surgery, demonstrating absent mass, with a small amount of scar tissue in the posterior abdominal wall. Frank ballooning of the oblique muscles, denervation atrophy and thinning of the 3 oblique (external oblique, internal oblique, and transverse abdominis) muscles, and thinning of the rectus abdominis muscle were present.

in the posterior abdominal wall penetrating through the quadratus, underneath the 12th rib (Fig. 2).

Abdominal CT and MRI after her lipoma surgery demonstrated an absent mass, with a small amount of scar tissue in the posterior abdominal wall. Frank ballooning of the oblique muscles, denervation atrophy and thinning of the 3 oblique (external oblique, internal oblique, and transverse abdominis) muscles, and thinning of the rectus abdominis muscle were demonstrated (Fig. 3).

\section{Hospital Course and Operation}

The patient was diagnosed with abdominal wall paresis due to axonotmetic injury to the subcostal nerve. All
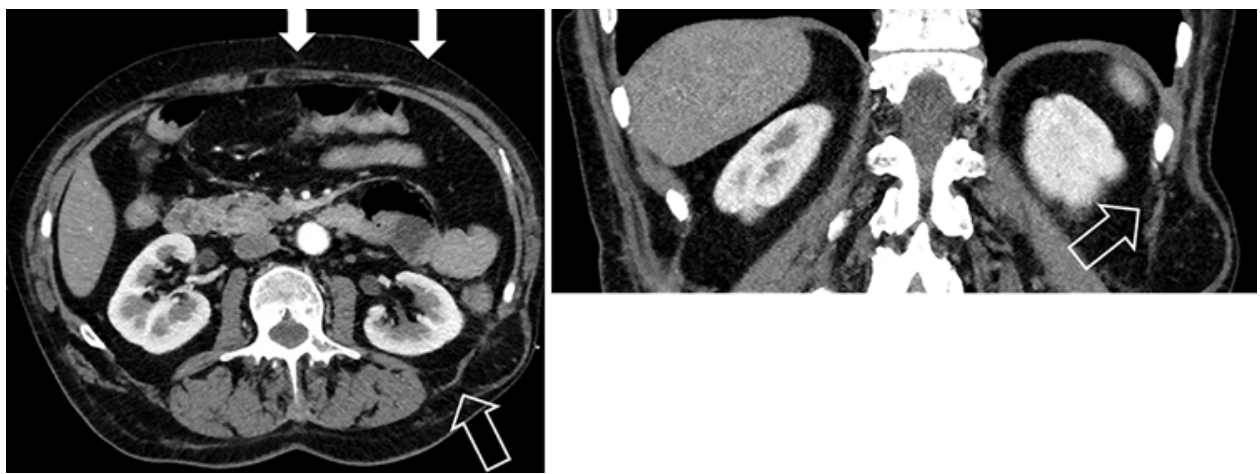

FIG. 2. Axial (left) and coronal (right) CT scans of the abdomen prior to the patient's lipoma surgery, demonstrating a lipoma-like mass (open arrow) in the posterior abdominal wall penetrating through the quadratus, underneath the 12th rib. Approximate location of laparoscopic port insertions indicated by solid arrows. 


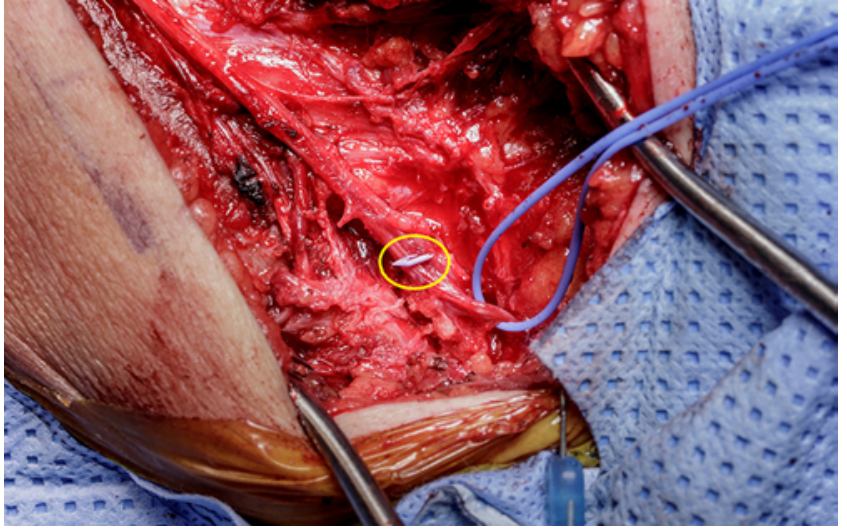

FIG. 4. Intraoperative photograph delineating a purple fragment of foreign material that looked like a harpoon (yellow circle) that was encased by the scarred portion of the nerve measuring $0.9 \times 0.2 \times 0.2 \mathrm{~cm}$ in diameter. Figure is available in color online only.

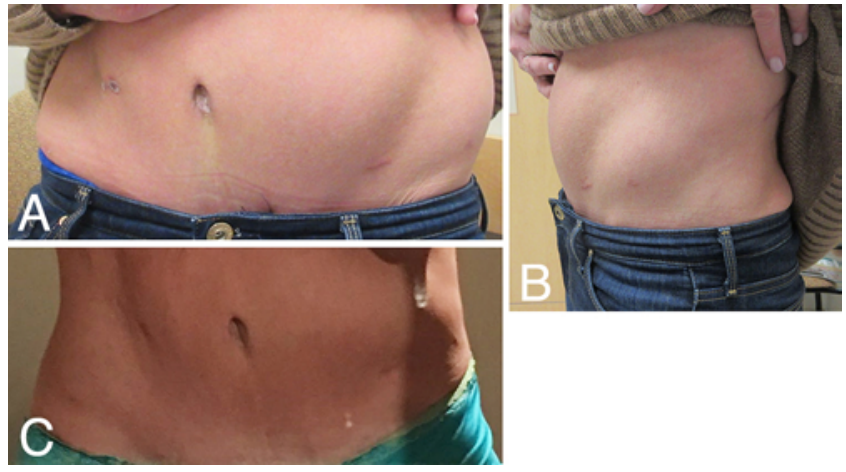

FIG. 5. A and B: Postoperative photographs at 3 months demonstrating return of tone in the lateral external oblique muscle and reduction in the pseudohernia and abdominal bulge. C: Postoperative photograph at 8 months demonstrating near-symmetrical curvature of the oblique muscles and contraction of the left rectus muscle. There is persistent deviation of the umbilicus, reflecting greater right-sided strength and excursion on abdominal wall contraction. Figure is available in color online only.

nerve repair. She was discharged to home on postoperative day 2. Three days later, she presented to the clinic for anesthetic catheter removal, where she noted substantial improvement in her pain and hypesthesia.

At the patient's 3-month follow-up, she described " $90 \%$ improvement" in flank wall pain. Her physical examination was notable for improved tone in the lateral oblique muscles (Fig. 5A and B). She described excellent contraction of the lateral oblique muscles to superficial electrical stimulation but no contraction of the rectus abdominis muscles to electrical stimulation.

At her 8-month follow-up, she had experienced substantial improvement in the ability to contract the oblique and rectus muscles (Fig. 5C); however, she continues to have deficits in maintaining resting tone. Ultrasound demonstrated good excursion of all 3 lateral abdominal wall muscles, with continued thickness asymmetry between the noninjured right side and the recovering left side (Table 1). Postoperative EMG at 8 months demonstrated unsustained fibrillation potentials, consistent with mild acute denervation, as well as minimally reduced recruitment compared with the contralateral side, consistent with moderate reinnervation.

\section{Discussion}

Laparoscopic surgery has revolutionized the surgical approaches to abdominal, thoracic, and spinal disease. It has shown superior results in terms of length of stay, blood loss, cosmesis, and complications compared with open approaches to similar pathologies., ${ }^{4,51,13}$ However, laparoscopic surgery brings its own, unique set of complications, particularly if used for expanded indications. Laparoscopic surgery has been associated with organ perforation, vessel injury, wound complications, and limited exposure. ${ }^{4,5,12,13}$ Although subcostal nerve injury following open surgery, ${ }^{9}$ and even minimally invasive spine surgery ${ }^{15}$ is a recognized complication, its occurrence after laparoscopic surgery is extremely uncommon. Injury 
TABLE 1. Postoperative ultrasound 8 months after nerve reconstruction comparing resting muscle thickness and contraction between the noninjured right side and the recovering left side

\begin{tabular}{ccccc}
\hline & \multicolumn{4}{c}{ Muscle } \\
\cline { 2 - 5 } Side & $\begin{array}{c}\text { Rectus } \\
\text { Abdominis }\end{array}$ & $\begin{array}{c}\text { External } \\
\text { Abdominal } \\
\text { Oblique }\end{array}$ & $\begin{array}{c}\text { Internal } \\
\text { Abdominal } \\
\text { Oblique }\end{array}$ & $\begin{array}{c}\text { Transverse } \\
\text { Abdominis }\end{array}$ \\
\hline Left & & & \\
\hline Rest & Not tested & $0.57 \mathrm{~cm}$ & $0.41 \mathrm{~cm}$ & $0.55 \mathrm{~cm}$ \\
\hline Contracted & $0.32 \mathrm{~cm}$ & $0.71 \mathrm{~cm}$ & $0.79 \mathrm{~cm}$ & $0.50 \mathrm{~cm}$ \\
\hline Right & & & & \\
\hline Rest & Not tested & $0.75 \mathrm{~cm}$ & $0.90 \mathrm{~cm}$ & Not tested \\
\hline Contracted & $1.08 \mathrm{~cm}$ & Not tested & $1.47 \mathrm{~cm}$ & Not tested \\
\hline Comparison & & & & \\
\hline Rest & - & $76 \%$ & $46 \%$ & - \\
\hline Contracted & $30 \%$ & - & $54 \%$ & - \\
\hline
\end{tabular}

- = not applicable.

to the intercostal/subcostal nerves leads to weakness and possible atrophy of the abdominal muscles. This results in a pseudohernia (abdominal bulge) formation, which can be aesthetically devastating for patients.

Most reports of subcostal nerve injury after laparoscopic surgery come from the urology literature. Crouzet et al. ${ }^{5}$ compared their experience with laparoscopic and open partial nephrectomy in 100 patients. The authors reported a statistically significant advantage in pseudohernia rate, preservation of abdominal wall muscle volume, and radiographic extent of flank bulge among their laparoscopic group. They found that only $12 \%$ of the patients treated laparoscopically noted a bulge, whereas $57 \%$ of patients treated with open surgery reported a permanent flank bulge.

Other scarce case reports in the literature have delineated the occurrence of subcostal nerve injury after laparoscopic cholecystectomy, laparoscopic appendectomy, splanchnic nerve radiofrequency ablation, and rib fractures. ${ }^{2,6,10,14,16}$ To the best of our knowledge, this is the first case of iatrogenic subcostal nerve injury following laparoscopic posterior abdominal wall lipoma resection. The challenge, of course, is that the use of an anteriorly placed laparoscope places the subcostal nerve behind the lipoma, where the surgeon is blind to it. In our review of the CT scan obtained prior to the lipoma surgery, we noted that the lipoma was situated directly at the anticipated course of the subcostal nerve-it was fortunate that the nerve was not resected along with the lipoma. Therefore, it is essential to understand the anatomy and course of the subcostal nerve to avoid injury and the development of pseudohernia among patients undergoing laparoscopic procedures.

Inferiorly to superiorly, the subcostal nerve runs with the subcostal vein and the artery in the inferior aspect of the 12th rib., The subcostal nerve innervates the rectus abdominis, the transversus abdominis, and the inferior portion of the external oblique muscles. ${ }^{1,7}$ In their comprehensive cadaveric and electrophysiological study, Fahim et al. ${ }^{7}$ demonstrated that the 11th intercostal and 12th sub- costal nerves are the predominant innervation to the anterolateral abdominal musculature. The authors reported 3 anatomical zones along the course of these nerves and proposed mechanisms for nerve injury during surgery. Zone 1 includes the portion of the nerves that travels in the intercostal groove. Zone 2 includes the segment of the 11th intercostal nerve traveling along the distal-most part of the 11th rib and the segment of the 12th subcostal nerve just beyond the tip of the 12th rib. Zone 3 includes the segment of the nerves curving anteroinferiorly around the abdominal wall. Retractors placed on the lower ribs might cause zone 1 injuries from crush or prolonged ischemia, whereas zone 3 injuries may be a result of transection injuries from port placement or wide flank incision. In our case, the injury was in zone 2, where the subcostal nerve separates from the protection of the 12 th rib.

It is therefore of paramount importance to understand the course of the subcostal nerve and the potential location(s) of nerve injury during spinal procedures in the thoracolumbar region. Grunert et al. ${ }^{8}$ examined the probability of injuring the lumbar plexus and its branches during the lateral transpsoas approach to the lumbar spine in 15 cadavers (26 sides). The authors reported two partial transections of the subcostal during these lateral fusion procedures. Similarly, Cahill and colleagues ${ }^{3}$ reported that $4.2 \%(n=5)$ of 118 patients (201 lumbar levels) who had undergone lateral lumbar approach procedures developed postoperative abdominal flank bulge. These injuries can occur during blunt dissection, causing direct transection, from the heat of electrocautery, or during closure of the abdominal musculature., 37,8 Additionally, spinal procedures that involve an L2 corpectomy may require the resection of the T12 rib, during which meticulous dissection is required to avoid iatrogenic injury to the subcostal nerve.

The prognosis of spontaneous nerve recovery depends on the severity of injury, and spontaneous recovery of subcostal nerve function has been previously reported..$^{11,16}$ Conservative management of these injuries is initially recommended. However, if progressive improvement in muscle tone does not occur within 3 months or if transection is suspected, surgical intervention might be advisable. We believe that careful identification of the partial injury within the nerve-allowing for repair of the injured fascicles while maintaining those in continuity-provided for early reduction in flank pain in our patient. Progressive return of muscle strength and tone appears to require axonal regeneration, as hallmarked by return of lateral tone in our patient at 3 months but no recovery of the anterior oblique and rectus abdominis muscles at this time.

\section{Acknowledgments}

We thank Kristin Kraus, MSc, for editorial assistance with this paper.

\section{References}

1. Alonso F, Graham R, Rustagi T, Drazin D, Loukas M, Oskouian RJ, et al: The subcostal nerve during lateral approaches to the lumbar spine: an anatomical study with relevance for injury avoidance and postoperative complications such 
as abdominal wall hernia. World Neurosurg 104:669-673, 2017

2. Butensky AM, Gruss LP, Gleit ZL: Flank pseudohernia following posterior rib fracture: a case report. J Med Case Reports 10:273, 2016

3. Cahill KS, Martinez JL, Wang MY, Vanni S, Levi AD: Motor nerve injuries following the minimally invasive lateral transpsoas approach. J Neurosurg Spine 17:227-231, 2012

4. Coccolini F, Catena F, Pisano M, Gheza F, Fagiuoli S, Di Saverio S, et al: Open versus laparoscopic cholecystectomy in acute cholecystitis. Systematic review and meta-analysis. Int J Surg 18:196-204, 2015

5. Crouzet S, Chopra S, Tsai S, Kamoi K, Haber GP, Remer EM, et al: Flank muscle volume changes after open and laparoscopic partial nephrectomy. J Endourol 28:1202-1207, 2014

6. Dellon AL: Intercostal neuroma pain after laparoscopic cholecystectomy: diagnosis and treatment. Plast Reconstr Surg 133:718-721, 2014

7. Fahim DK, Kim SD, Cho D, Lee S, Kim DH: Avoiding abdominal flank bulge after anterolateral approaches to the thoracolumbar spine: cadaveric study and electrophysiological investigation. J Neurosurg Spine 15:532-540, 2011

8. Grunert P, Drazin D, Iwanaga J, Schmidt C, Alonso F, Moisi $\mathrm{M}$, et al: Injury to the lumbar plexus and its branches after lateral fusion procedures: a cadaver study. World Neurosurg 105:519-525, 2017

9. Guastella V, Mick G, Soriano C, Vallet L, Escande G, Dubray $\mathrm{C}$, et al: A prospective study of neuropathic pain induced by thoracotomy: incidence, clinical description, and diagnosis. Pain 152:74-81, 2011

10. Korenkov M, Rixen D, Paul A, Köhler L, Eypasch E, Troidl $\mathrm{H}$ : Combined abdominal wall paresis and incisional hernia after laparoscopic cholecystectomy. Surg Endosc 13:268269, 1999

11. Montagna P, Medori R, Liguori R, Cortelli P: Abdominal neuropathy after renal surgery. Ital J Neurol Sci 6:357-358, 1985

12. Riviere D, Gurusamy KS, Kooby DA, Vollmer CM, Bes- selink MG, Davidson BR, et al: Laparoscopic versus open distal pancreatectomy for pancreatic cancer. Cochrane Database Syst Rev 4:CD011391, 2016

13. Sajid MS, Khawaja AH, Sains P, Singh KK, Baig MK: A systematic review comparing laparoscopic vs open adhesiolysis in patients with adhesional small bowel obstruction. Am J Surg 212:138-150, 2016

14. Tewari S, Agarwal A, Gautam SK, Madabushi R: Intercostal neuralgia occurring as a complication of splanchnic nerve radiofrequency ablation in a patient with chronic pancreatitis. Pain Physician 20:E747-E750, 2017

15. Uribe JS, Dakwar E, Cardona RF, Vale FL: Minimally invasive lateral retropleural thoracolumbar approach: cadaveric feasibility study and report of 4 clinical cases. Neurosurgery 68 (1 Suppl Operative):32-39, 2011

16. van Ramshorst GH, Kleinrensink GJ, Hermans JJ, Terkivatan T, Lange JF: Abdominal wall paresis as a complication of laparoscopic surgery. Hernia 13:539-543, 2009

\section{Disclosures}

The authors report no conflict of interest concerning the materials or methods used in this study or the findings specified in this paper.

\section{Author Contributions}

Conception and design: both authors. Acquisition of data: AbouAl-Shaar. Analysis and interpretation of data: both authors. Drafting the article: Abou-Al-Shaar. Critically revising the article: both authors. Reviewed submitted version of manuscript: both authors. Approved the final version of the manuscript on behalf of both authors: Mahan.

\section{Correspondence}

Mark A. Mahan: University of Utah, Salt Lake City, UT. mark.mahan@hsc.utah.edu. 